\title{
Neurologic and Renal Outcomes of Elective Proximal Aortic Repair and Current Cannulation Trends
}

\author{
Sahin Iscan* and Bortecin Eygi \\ Department of Cardiovascular Surgery, Katip Celebi University Izmir Ataturk Education and Research \\ Hospital, Izmir, Turkey
}

*Corresponding author: Sahin Iscan, Department of Cardiovascular Surgery, Katip Celebi University Izmir Ataturk Education and Research Hospital, 35360, Izmir, Turkey. Tel: 00905054882090

\begin{abstract}
Background: We aimed to determine factors influencing neurologic dysfunction and acute kidney injury (AKI) in patients undergoing elective aortic aneurysm repair and we also investigated them according to cannulation sites.

Methods: From January 2012 to April 2018, 112 patients received proximal aortic repair. We used aortic arch, axillary and femoral artery cannulation. Antegrade cerebral protection and total circulatory arrest were used.

Results: We detected 6 (5.4\%) transient and 6 (5.4\%) permanent neurologic dysfunction. Peripheral arterial diseases, total bypass time, transfusion and AKI showed correlation with neurologic dysfunction. Peripheral arterial diseases and AKI were independent risk factors $(p<0.05)$. AKI was reported in 38 patients. Postoperative revision, cross-clamp time, total bypass time and total circulatory arrest showed correlation with AKI $(p<0.05)$. Patients with neurologic dysfunction (66\%) and patients with AKI (63\%) had more femoral cannulation.

Conclusion: Proximal aortic repair can be achieved with low morbidity and mortality using appropriate cerebral protection and cannulation technique. Neurologic dysfunction and $\mathrm{AKI}$ are important factors for aortic surgery and femoral cannulation has the highest risk for neurologic dysfunction and $\mathrm{AKI}$.
\end{abstract}

\section{Keywords}

Aortic aneurysm, Neurologic dysfunction, Kidney injury, Cannulation

\section{Introduction}

Cerebral protection is a big technical difficulty for aortic surgery. Increased use of antegrade cerebral perfusion (ACP) and deep hypotermic circulatory arrest in experienced hands made open surgery of the ascending aorta and aortic arch aneurysms more successful today. The increase in the variety of surgical equipment to provide unilateral and bilateral cerebral perfusion or monitorization, the effectiveness of the cerebral protection instantly decreased the risk of perioperative stroke or postoperative morbidity and mortality. Despite these improvements, there are many problems which affect patients' prognosis, like arterial cannulation strategies, cross clamp time, cardiopulmonary bypass time and patient related co-morbidity factors [1,2].

Although we know that the best choice for providing arterial perfusion to patients during cardiopulmonary bypass is the antegrade flow via aortic cannulation, we also need another arterial perfusion sites to perform aortic hemiarch and total arch surgery. But flow dynamics in these arterial cannulation sites (axillary and femoral arterial sites), and patient related co-morbidities (variations of the circle of Willis, intracerebral arterial pathologies, abdominal aortic thrombi and peripheral arterial diseases (PAD)), still have the complication risk for brain, kidneys and other organs. Today, stroke rates of the patients are related to prolonged total circulatory arrest (TCA) or incomplete cerebral protection [2]. Multiorgan deficiencies are related to prolonged cardiopulmonary bypass or arterial perfusion which does not provide antegrade flow to these organs and thus leading to deficient organ perfusion, especially on brain and kidneys [2-4]. So, despite increased experience in surgical technique and improved brain protection strategies, there are still problems to be solved.

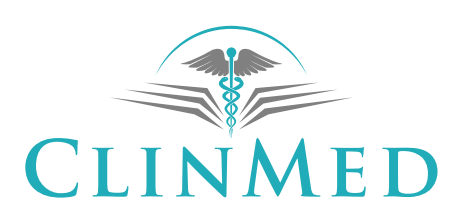

INTERNATIONAL LIBRARY
Citation: Iscan S, Eygi B (2018) Neurologic and Renal Outcomes of Elective Proximal Aortic Repair and Current Cannulation Trends. Int J Clin Cardiol 5:128. doi.org/10.23937/2378-2951/1410128

Accepted: November 05, 2018; Published: November 07, 2018

Copyright: (c) 2018 Iscan S, et al. This is an open-access article distributed under the terms of the Creative Commons Attribution License, which permits unrestricted use, distribution, and reproduction in any medium, provided the original author and source are credited. 
In this advanced era of the open aortic aneursym surgery, we wanted to present our single centre experience. So, we retrospectively analyzed our elective aortic root, ascending aorta and aortic hemiarch surgery experiences in concomitant with aortic valve diseases. We investigated them for postoperative outcomes such as neurologic dysfunction and kidney injury relating to arterial cannulation sites.

\section{Materials and Methods}

Between January 2012 and April 2018, 112 consecutive patients, who underwent elective aortic root, ascending aorta and aortic hemiarch operations, were retrospectively analyzed. All of the operations were the first cardiac surgery for the patients. The main indications for aortic operations were degenerative aortic aneurysm and aortic valve disease, but mitral valve surgery and coronary artery bypass surgery were added to the procedure in the presence of concomitant diseases. None of the patients had preexisting neurologic injury.

All patients were operated on using midsternal approach. We used aortic arch, axillary artery and femoral artery for arterial cannulation and a venous single twostage cannula in the right atrium in the majority of the patients. 8-Fr Dacron graft was anastomosed to axillary artery in an end-to-side fashion for axillary cannulation. Appropriate femoral arterial cannula was inserted through retrograde way into common femoral artery using Seldinger technique for femoral cannulation. We did not use femoral artery when the presence of abdominal aortic thrombi or aneurysm on computed tomography. In the TCA cases, patients were profoundly cooled to a core temperature of less than $18^{\circ} \mathrm{C}$ and external cooling was performed to the head with ice packing. In the ACP cases, patients were cooled to a core temperature between $22^{\circ} \mathrm{C}$ and $25^{\circ} \mathrm{C}$, followed by systemic arrest and declamping of the aorta. ACP was performed through antegrade way. In patients with axillary cannulation, brachiocephalic artery was clamped before removing the aortic clamp and the cerebral perfusion was initiated at a rate of $10 \mathrm{~mL} / \mathrm{min} / \mathrm{kg}$. In patients with femoral artery cannulations, ACP was performed with a cannula which was inserted through the ostium of the brachiocephalic artery. Bilateral carotid arterial perfusion was performed according to preference of surgeon, but cerebral saturation measurement and arterial pressure was the main indicator to determine the cerebral perfusion method in these patients. Intraoperative cerebral monitoring was achieved by oxygen saturation in the frontal lobes with near-infrared spectroscopy and right radial artery pressure lines. Aortic aneurysms which extend to proximal of the aortic arch, short curvature of the aortic arch was excised and the graft was anastomosed in a hemiarch configuration. Hemiarch reconstruction was achieved using ACP or TCA with a distal open aortic technique. In the majority of the patients, after completing hemiarch anastomosis, arterial cannula was transferred to the graft to provide antegrade perfusion for the rest of the procedure.

Postoperative confusion, agitation, obtundation and delirium were accepted as temporary neurologic dysfunction. Stroke, coma and presence of new brain lesions confirmed by imaging studies (Computed tomography, magnetic resonance imaging) were defined as permanent neurologic dysfunction [5]. AKI was defined using Acute Kidney Injury Network Criteria. These criteria stage kidney injury according to the ratio of postoperative serum creatinin to preoperative level; 1.5 to 2 times: Stage 1, 2 to 3 times: Stage 2, 3 times: Stage 3 [6].

\section{Statistical Analysis}

Continuous variables were expressed as mean \pm standard deviation and categorical variables as numbers and percentages. Univariate and multivariable logistic regression analyses were performed to determine risk factors of neurologic dysfunction and AKI. Statistical analysis was performed using IBM SPSS Statistics, version 22 (IBM Corp, Armonk, NY).

\section{Results}

112 consecutive patients, who underwent elective aortic root, ascending aorta and aortic hemiarch operations, were retrospectively analyzed. There were 68 male patients $(60.7 \%)$ and 44 female patients (39.3\%), with a mean age of $58.3 \pm 14$ years. 36 patients underwent Bentall operation (composite graft replacement of the aortic valve, aortic root and ascending aorta, with re-implantation of the coronary arteries into the graft), 32 patients underwent ascending aorta replacement and 44 patients underwent ascending aorta and aortic valve replacement. Aortic resection was extended to aortic hemiarch in 38 patients. 8 patients had mitral valve replacement and 7 patients had coronary bypass surgery additionally to main aneurysm surgery. All preoperative data are depicted in Table 1.

Table 1: Demographic data of the patients.

\begin{tabular}{|c|c|c|c|}
\hline \multicolumn{2}{|c|}{ Variable } & $\mathbf{N}(\%)$ & Mean \pm SD \\
\hline \multicolumn{2}{|c|}{ Age } & & $58.3 \pm 14$ \\
\hline \multirow{2}{*}{\multicolumn{2}{|c|}{ Sex }} & Male $68(60.7 \%)$ & \\
\hline & & Female $44(39.3 \%)$ & \\
\hline \multicolumn{2}{|c|}{ Diabetes Mellitus } & $63(56.3 \%)$ & \\
\hline \multicolumn{2}{|c|}{ Hypertension } & $84(75 \%)$ & \\
\hline \multicolumn{2}{|c|}{ PAD } & $29(25.9 \%)$ & \\
\hline \multicolumn{2}{|c|}{ COPD } & $57(50.9 \%)$ & \\
\hline \multicolumn{3}{|c|}{ BMI } & $27.8 \pm 6$ \\
\hline \multirow{3}{*}{$\mathrm{BMI}$} & $<25$ & $35(31.3 \%)$ & \\
\hline & $25-30$ & $44(39.3 \%)$ & \\
\hline & $<30$ & $33(29.5 \%)$ & \\
\hline \multicolumn{2}{|l|}{ EF } & & $55.7 \pm 7.6$ \\
\hline \multicolumn{2}{|c|}{ Preoperative creatinin (mg/dl) } & & $0.9 \pm 0.4$ \\
\hline \multicolumn{2}{|c|}{ Preoperative HGB (g/L) } & & $12.5 \pm 3.6$ \\
\hline
\end{tabular}

SD: Standard deviation; PAD: Peripheral arterial disease; COPD: Chronic obstructive pulmonary disease; BMI: Body mass index; EF: Echocardiographic ejection fraction; HGB: Hemoglobin. 
Table 2: Operative data.

\begin{tabular}{|c|c|c|c|}
\hline \multicolumn{2}{|l|}{ Variable } & $\mathbf{N}(\%)$ & Mean/SD \\
\hline \multirow{3}{*}{$\begin{array}{l}\text { Cardiac } \\
\text { operation }\end{array}$} & Bentall & $36(32.2 \%)$ & \\
\hline & AVR + AAR & $44(39.3 \%)$ & \\
\hline & AAR & $32(28.5 \%)$ & \\
\hline \multirow{2}{*}{$\begin{array}{l}\text { Aortic distal } \\
\text { anastomosis }\end{array}$} & Ascending aorta & $74(67 \%)$ & \\
\hline & Hemiarch & $38(33 \%)$ & \\
\hline \multirow{3}{*}{ Cannulation } & Axillary & $20(17.8 \%)$ & \\
\hline & Arcus & $50(44.6 \%)$ & \\
\hline & Femoral & $42(37.6 \%)$ & \\
\hline \multicolumn{3}{|l|}{ CCT (min) } & $89.2 \pm 37.2$ \\
\hline \multicolumn{3}{|l|}{ TBT (min) } & $127.2 \pm 48.6$ \\
\hline \multicolumn{2}{|l|}{$\mathrm{ACP} / \mathrm{min}$} & $26(23.2 \%)$ & $20.2 \pm 9.4$ \\
\hline \multicolumn{2}{|l|}{$\mathrm{TCA} / \mathrm{min}$} & $12(10.7 \%)$ & $16.1 \pm 7.6$ \\
\hline
\end{tabular}

SD: Standard deviation; AVR: Aortic valve replacement; AAR: Ascending aorta replacement; CCT: Cross clamp time; TBT: Total bypass time; ACP: Antegrade cerebral perfusion; TCA: Total circulatory arrest.
Table 3: Postoperative complications.

\begin{tabular}{|c|c|c|c|}
\hline \multicolumn{2}{|c|}{ Variable } & N (\%) & Mean/SD \\
\hline \multicolumn{2}{|c|}{ Postoperative drainage (ml) } & & $632.1 \pm 382.5$ \\
\hline \multicolumn{2}{|c|}{ RBC Transfusion (u) } & & $1.8 \pm 1.5$ \\
\hline \multicolumn{2}{|c|}{ Inotropic support } & $66(58.9 \%)$ & \\
\hline \multicolumn{2}{|c|}{ Extubation time (hour) } & & $20.3 \pm 15.5$ \\
\hline \multicolumn{2}{|c|}{ Postoperative creatinin (mg/dl) } & & $1.2 \pm 0.7$ \\
\hline \multirow{3}{*}{ AKI } & Stage I & $25(22.3 \%)$ & \\
\hline & Stage II & $12(10.7 \%)$ & \\
\hline & Stage III & $1(0.9 \%)$ & \\
\hline \multicolumn{2}{|c|}{ Dialysis } & $1(0.9 \%)$ & \\
\hline \multicolumn{2}{|c|}{ TND } & $6(5.4 \%)$ & \\
\hline \multicolumn{2}{|l|}{ PND } & $6(5.4 \%)$ & \\
\hline \multicolumn{2}{|c|}{ In-hospital death } & $9(8 \%)$ & \\
\hline \multicolumn{2}{|c|}{ ICU stay (day) } & & $4.7 \pm 3.3$ \\
\hline \multicolumn{2}{|c|}{ Total hospital stay (day) } & & $11.3 \pm 5.6$ \\
\hline
\end{tabular}

SD: Standard deviation; RBC: Red blood cell; AKI: Acute kidney injury; TND: Temporary neurologic dysfunction; PND: Permanent neurologic dysfunction; ICU: Intensive care unit.

Table 4: Univariate and multivariate analysis for neurologic dysfunction.

\begin{tabular}{|c|c|c|c|c|c|c|c|c|c|c|}
\hline \multirow{3}{*}{\begin{tabular}{|l} 
Variable \\
Age
\end{tabular}} & \multicolumn{5}{|c|}{ Univariate } & \multicolumn{5}{|c|}{ Multivariate } \\
\hline & \multirow{2}{*}{\begin{tabular}{|l|} 
OR \\
1.07
\end{tabular}} & \multicolumn{3}{|c|}{$\% 95 \mathrm{Cl}$} & \multirow{2}{*}{\begin{tabular}{|l}
$p$ \\
0.028
\end{tabular}} & \multirow[t]{2}{*}{ OR } & \multicolumn{3}{|c|}{$\% 95 \mathrm{Cl}$} & \multirow[t]{2}{*}{ p } \\
\hline & & 1.01 & - & 1.13 & & & & & & \\
\hline Sex & 1.33 & 0.38 & - & 4.72 & 0.656 & & & & & \\
\hline BMI & 0.98 & 0.88 & - & 1.08 & 0.662 & & & & & \\
\hline EF & 1.00 & 0.93 & - & 1.09 & 0.937 & & & & & \\
\hline HT & 4.07 & 0.50 & - & 33.03 & 0.189 & & & & & \\
\hline DM & 1.10 & 0.33 & - & 3.70 & 0.878 & & & & & \\
\hline COPD & 5.64 & 1.18 & - & 27.05 & 0.031 & & & & & \\
\hline PAD & 12.00 & 2.97 & - & 48.44 & 0.000 & 17.43 & 3.37 & - & 90.3 & 0.001 \\
\hline Revision & 2.76 & 1.30 & - & 5.86 & 0.008 & & & & & \\
\hline CCT & 1.01 & 1.00 & - & 1.03 & 0.185 & & & & & \\
\hline TBT & 1.01 & 1.00 & - & 1.02 & 0.031 & & & & & \\
\hline ACP time & 2.69 & 0.77 & - & 9.33 & 0.120 & & & & & \\
\hline TCA time & 0.74 & 0.09 & - & 6.26 & 0.779 & & & & & \\
\hline Drainage & 1.00 & 1.00 & - & 1.00 & 0.283 & & & & & \\
\hline Inotropic support & $>100$ & 0.00 & - & $>100$ & 0.997 & & & & & \\
\hline RBC Transfusion & 1.67 & 1.18 & - & 2.37 & 0.004 & & & & & \\
\hline AKI & 29.74 & 3.66 & - & 241.46 & 0.001 & 41.41 & 4.40 & - & 389.8 & 0.001 \\
\hline Dialysis & 4.45 & 0.37 & - & 53.20 & 0.238 & & & & & \\
\hline ICU stay & 1.20 & 1.04 & - & 1.38 & 0.014 & & & & & \\
\hline In hospital stay & 0.86 & 0.72 & - & 1.04 & 0.119 & & & & & \\
\hline
\end{tabular}

Logistic Regression Test: BMI: Body mass index; EF: Ejection fraction; HT: Hypertension; DM: Diabetes mellitus; COPD: Chronic obstructive pulmonary disease; PAD: Peripheral arterial disease; CCT: Cross clamp time; TBT: Total bypass time; ACP: Antegrade cerebral perfusion; TCA: Total circulatory arrest; RBC: Red blood cell; ICU: Intensive care unit.

We used aortic arch cannulation site in 50 patients (44.6\%), axillary artery cannulation site in 20 patients (17.8\%) and femoral artery cannulation site in 42 patients (37.6\%) for the institution of CPB. Cerebral protection was achieved by means of ACP in 26 patients (23.2\%). TCA was used in 12 patients (10.7\%). Aortic resection was limited to ascending aorta in 74 patients $(67 \%)$ and it was extended to aortic hemiarch in 38 patients (33\%). The mean aortic cross-clamp time and total bypass time were $89.2 \pm 37.2$ minutes and $127.2 \pm$ 48.6 minutes, respectively. The mean duration of ACP and TCA were $20.2 \pm 9.4$ minutes and $16.1 \pm 7.6 \mathrm{~min}$ utes, respectively (Table 2 ).
The mean postoperative first day drainage was 632.1 $\pm 385.5 \mathrm{ml}$. 14 patients needed to early postoperative reoperation (revision) due to bleeding problems. Mean transfused red blood cell was $1.8 \pm 1.5$ units. 66 (58.9\%) patients needed to positive inotropic treatment (Dopamine, dobutamine or adrenaline) on postoperative period due to hypotension or low cardiac output. Mean intensive care unit stay was $4.7 \pm 3.3$ days and mean in hospital stay was $11.3 \pm 5.6$ days. Nine patients $(8 \%)$ died during hospitalization (Table 3 ).

\section{Neurologic dysfunction}

TND and PND was reported in 6 (5.4\%) and 6 (5.4\%) 
patients respectively. At univariate analysis, age $(\mathrm{p}=$ $0.028)$, chronic obstructive pulmonary disease (COPD) $(p=0.031), \operatorname{PAD}(p<0.001)$, postoperative revision ( $p$ $=0.008)$, total bypass time $(p=0.031)$, red blood cell transfusion $(p=0.004)$ and AKI $(p=0.001)$ showed a statistically significant correlation with neurologic dysfunction. At multivariate analysis, PAD ( $p=0.001)$ and AKI $(p=0.001)$ showed a significant correlation with neurologic dysfunction (Table 4). We saw that patients with neurologic dysfunction had more femoral cannulation $(\mathrm{n}=8,66 \%)$.

\section{Kidney Injury}

Acute kidney injury was reported in 38 (33.9\%) patients (Stage I $n=25$, Stage II $n=12$ and Stage III $n=$ 1). 1 patients $(0.9 \%)$ needed dialysis on postoperative period. At univariate analysis, postoperative revision ( $p$ $=0.019)$, cross clamp time $(p=0.036)$, total bypass time $(p=0.026)$, TCA $(p=0.018)$, postoperative drainage $(p=$ $0.036)$ and red blood cell transfusion $(p=0.002)$ showed a significant correlation with AKI. At multivariate analysis, there was no significant correlation with the variables (Table 5). We saw that femoral cannulation site was used more frequently in patients with AKI ( $n=24$, $63 \%)$. Patients who had stage II and III AKI ( $n=10,76 \%)$ had more femoral cannulation ratio compared to patients with stage I AKI $(n=14,56 \%)$.

\section{Discussion}

Nowadays there is a wide experience in the treatments of ascending aortic aneurysms and aortic valve diseases in many heart surgery centers. Although, highly experienced aortic surgeons are still facing contro- versies in surgical approaches extending to the aortic arch. One of the most important intraoperative factors affecting the surgical morbidity of these patients is how the cannulation is performed and how the arterial flow is provided and how the cerebral protection is maintained. The strategy of cannulation and the strategy of cerebral protection, which are created as a result of patient-specific approaches in experienced centers, are the leading determinants of postoperative neurological damage, AKI and death $[7,8]$. In this study, we investigated the postoperative neurological dysfunction, AKI and perioperative factors affecting patients with chronic ascending and aortic arch aneurysms after elective surgery. We also discussed in detail the techniques of cannulation sites used in patients with neurological dysfunction and AKI.

The most important factors that determine the risk of neurological dysfunction in aneurysm surgery are how cerebral protection is achieved and where the cannulation is. Despite the fact that ACP and TCA applied under deep hypothermic circulatory arrest, they are the two most frequently used cerebral protection techniques, the use of TCA in elective aneurysm surgery has decreased over time. TCA largely left its place in unilateral and bilateral ACP applied in deep hypothermia [9]. In this study, we performed aortic hemiarch replacement in 38 patients (33\%) and performed cerebral protection (ACP $n=26,23.3 \%$, TCA $n=12,10.7 \%$ ). The use of unilateral and bilateral ASCP technique under deep hypothermic arrest is increasing while our TCA practice to decline over the years. Postoperative TND rate was $5.4 \%(n=6)$ and PND rate was $5.4 \%(n=6)$. In a

Table 5: Univariate and multivariate analysis for AKI.

\begin{tabular}{|c|c|c|c|c|c|c|c|c|}
\hline \multirow{3}{*}{$\begin{array}{l}\text { Variables } \\
\text { Age }\end{array}$} & \multicolumn{5}{|c|}{ Univariate } & \multicolumn{3}{|c|}{ Multivariate } \\
\hline & \multirow{2}{*}{\begin{tabular}{|l|} 
OR \\
1.03 \\
\end{tabular}} & \multicolumn{3}{|c|}{$\% 95 \mathrm{Cl}$} & \multirow{2}{*}{\begin{tabular}{|l}
$\mathbf{p}$ \\
0.098
\end{tabular}} & \multirow[t]{2}{*}{ OR } & \multirow[t]{2}{*}{$\% 95 \mathrm{Cl}$} & \multirow[t]{2}{*}{ p } \\
\hline & & 1.00 & - & 1.06 & & & & \\
\hline Sex & 1.65 & 0.72 & - & 3.76 & 0.233 & & & \\
\hline BMI & 0.96 & 0.90 & - & 1.03 & 0.237 & & & \\
\hline EF & 0.98 & 0.93 & - & 1.03 & 0.422 & & & \\
\hline BMI & 0.96 & 0.90 & - & 1.03 & 0.237 & & & \\
\hline HT & 2.26 & 0.83 & - & 6.16 & 0.112 & & & \\
\hline DM & 0.94 & 0.43 & - & 2.07 & 0.880 & & & \\
\hline COPD & 1.80 & 0.81 & - & 3.99 & 0.146 & & & \\
\hline PAD & 1.55 & 0.65 & - & 3.70 & 0.327 & & & \\
\hline Revision & 2.04 & 1.12 & - & 3.72 & 0.019 & & & \\
\hline CCT & 1.01 & 1.00 & - & 1.02 & 0.036 & & & \\
\hline TBT & 1.01 & 1.00 & - & 1.02 & 0.026 & & & \\
\hline ACP time & 0.65 & 0.25 & - & 1.73 & 0.391 & & & \\
\hline TCA time & 4.67 & 1.31 & - & 16.69 & 0.018 & & & \\
\hline Drainage & 1.00 & 1.00 & - & 1.00 & 0.036 & & & \\
\hline Inotropic support & 2.20 & 0.95 & - & 5.08 & 0.064 & & & \\
\hline RBC Transfusion & 1.59 & 1.19 & - & 2.13 & 0.002 & & & \\
\hline Dialysis & $>100$ & 0.00 & - & $>100$ & 0.999 & & & \\
\hline ICU stay & 1.15 & 1.01 & - & 1.30 & 0.034 & & & \\
\hline In hospital stay & 1.02 & 0.95 & - & 1.09 & 0.540 & & & \\
\hline
\end{tabular}

Logistic Regression Test: BMI: Body mass index; EF: Ejection fraction; HT: Hypertension; DM: Diabetes mellitus; COPD: Chronic obstructive pulmonary disease; PAD: Peripheral arterial disease; CCT: Cross clamp time; TBT: Total bypass time; ACP: Antegrade cerebral perfusion; TCA: Total circulatory arrest; RBC: Red blood cell; ICU: Intensive care unit. 
study conducted by Dhuranlar, et al. [10] involving 837 patients with elective, urgent and emergent ascending and aortic arch aneurysm, the PND was 2.3\%. In 501 patient undergoing aortic arch surgery performed by Khaladj, et al. [11], TND was $13.4 \%$ and PND was $9.6 \%$. In a study conducted by Cefarelli, et al. [4], in which 563 aortic hemiarch replacements and 44 total arch replacements were performed, the TND and PND ratios were reported as $5.3 \%$ and $6.2 \%$, respectively. Compared with these studies with high patient populations, we observed similar neurological dysfunction rates in our elective patients.

In univariate analysis, age, COPD, PAD, postoperative revision, $\mathrm{TBT}, \mathrm{RBC}$ transfusion and $\mathrm{AKI}$ are the most important factors in development neurological dysfunction. In multivariate analysis, PAD presence and postoperative AKI development were found to be an independent risk factor for neurological damage. When the patients with neurological damage were examined in detail regarding the location of the cannulation, it was observed that femoral cannulation was used in $66 \%$ of these patients $(n=8)$. Femoral artery cannulation and retrograde arterial perfusion increase the risk of cerebral emboli and increase the likelihood of having a neurological event. In our study, aortic arch cannulation was performed in $44.6 \%$, axillary artery cannulation in $17.8 \%$ and femoral artery cannulation in $37.6 \%$ of the patients. Our tendency to perform aortic arch cannulation or axillary artery cannulation as much as possible for the reasons mentioned has increased over the years. We strongly believe that, if possible, arterial perfusion with a cannula positioned at the distal end of the aneurysm or arterial perfusion with axillary arterial side graft anastomosis in aortic hemiarch and aortic arch surgery is the perfusion technique that minimizes the risk of cerebral damage and provides antegrade flow.

One of the other important factors affecting postoperative morbidity and mortality is AKI. In many studies investigating AKI associated with coronary bypass and heart valve surgery, the amount of blood transfusion and duration of cardiopulmonary bypass has been described as the most important factors influencing AKI $[12,13]$. In a study conducted by Lio, et al. [14] in 96 patients with aortic hemiarch and total aortic arch replacement, the rate of AKI was $22 \%$ and postoperative dialysis requirement was $1 \%$. The rate of AKI in the Japanese population who underwent total arch replacement performed by Okita, et al. [15] was $15.4 \%$ and the dialysis requirement was $7.7 \%$. In another study evaluating 890 patients who underwent only aortic root replacement, the dialysis requirement was reported as $0.5 \%$ [16]. The rate of patients requiring dialysis in our study is $0.9 \%(n=1)$ although the AKI ratio is higher than other studies (Stage I: $22.3 \%$, Stage II: $10.7 \%$ and Stage III: $10.9 \%$ ) depending on the method used to describe AKI. Our results seem compatible with other studies. In our study, univariate analysis revealed that as the cross clamp time, total bypass time, TCA, postoperative drainage, revisions, and RBC transfusion increased, AKI development also increased. A direct risk factor for multivariate analysis was not identified (Table 5). These results indicate that $A K I$ is closely related to cardiopulmonary bypass and transfusion same as in other cardiac surgical procedures.

We think that the surgical transfusion volume is influenced by the surgical variables such as the patient's preoperative condition, cardiopulmonary bypass time and postoperative revision need, but we do not think that the only risk factor is perfusion time related to cardiopulmonary bypass $[17,18]$. We evaluated the effects of axillary artery, ascending aorta, and femoral artery cannulations on the renal and carotid artery flows in a study that we had previously performed to investigate the relationship between organ perfusions and the cannulation sites. We conclude that the renal artery flows in the setting of axillary artery and ascending aorta cannulation is significantly higher than that obtained with femoral cannulation. In the results of this experimental study, there was no significant difference between renal artery flows with axillary and ascending aorta cannulation sites [17]. These results indicate that the failure of antegrade perfusion in addition to the atheroembolic risk associated with retrograde perfusion of femoral cannulation site has also a negative effect on organ perfusion. In our study, we observed that femoral cannulation rate among the AKI patients was $63 \%(n=$ 24). In addition, femoral cannulation is associated with development of higher rate of advanced kidney injury in patients with AKI (Stage II and III AKI, $n=10,76 \%$ ).

In an experimental study conducted by Gaier, et al. [18], carotid artery perfusions were examined during ECMO perfusion. In the absence of cardiac output, carotid artery flows in the setting of ascending aortic cannulation and femoral artery cannulation were found to be higher than those with axillary artery cannulation. Experimental work by Demertzis, et al. [19] also showed a decrease in carotid flow with axillary artery perfusion, and this decrease was attributed to Venturi effect. We have also found in our experimental study, the flow through the femoral cannulation cannot be transmitted to aortic arch with the increase of cardiac output. We also found that central cannulation and axillary cannulation had positive effects on intraabdominal organ perfusion and femoral cannulation was inadequate for intraabdominal circulation [17]. Gaier, et al. [18] found cerebral perfusion pressure to be at least as effective as the flow rate for cerebral circulation and indicated that the cerebral perfusion pressure was highest with ascending aorta and axillary artery cannulation. In another study, unilateral and bilateral cerebral perfusion were found to have no effect on mortality, but unilateral perfusion was reported to have high risk for neurological dysfunction due to variations of the circle of Willis, thus the bilateral perfusion may be more effective than 
the unilateral one [20]. These results show that for all organs, antegrade perfusion is necessary both during cardiopulmonary bypass and during cerebral perfusion. The most suitable pathway for cerebral and renal circulation in the light of these data is the ascending aortic cannulation and aortic arch cannulation. For cerebral perfusion, we also believe that unilateral cerebral perfusion with axillary cannulation may be less favorable due to Venturi effect, frequent variations of the circle of Willis and lower perfusion pressures. The insufficiency of this perfusion pressure can be avoided by bilateral perfusion of carotid arteries as far as possible, and our surgical tendency is changing that way over the years. In addition, for aneurysm surgery, regardless of the perfusion problems, we believe that atheroembolism, caused by femoral cannulation, is a very real risk factor and femoral cannulation site should be preferred only with certain exceptions. We conclude that systemic perfusion provided by aortic arch or axillary artery cannulation is the most appropriate route for cerebral and peripheral circulation $[4,21,22]$.

Given the retrospective nature of our study, the possibility of single-centered work and the number of patients has a lower level of evidence than multicenter prospective randomized trials with larger databases. Detection of preoperative cerebral circulation, detection of signs such as intraoperative ACP time and temperature, and inclusion of these results in an evaluable study may give more reliable results on ACP. It also would be better to compare patients who had circulatory arrest and those who didn't. But we could not do it due to low patient population. There is a need for more standardization.

In conclusion, ascending aorta, aortic root, and aortic arch surgery with appropriate surgical technique carry acceptable risk for neurological damage and kidney damage. There is a close relationship between the technique of cannulation and neurological dysfunction and development of AKI. Femoral cannulation adversely affects cerebral perfusion and intraabdominal perfusion, disrupting brain and kidney circulation and increasing mortality and morbidity rates. We conclude that the use of bilateral antegrade cerebral perfusion with axillary artery or aortic arch cannulation for ascending aortic and aortic arch surgery is the surgical technique that minimizes postoperative morbidity and mortality.

\section{Acknowledgement}

Authors have nothing to disclose with regard to financial or technical support.

\section{Conflict of Interest}

There is no conflict of interest between authors.

\section{References}

1. Williams JB, Peterson ED, Zhao Y, O'Brien SM, Andersen ND, et al. (2012) Contemporary results for proximal aortic replacement in North America. J Am Coll Cardiol 60: 1156-1162.
2. Halkos ME, Kerendi F, Myung R, Kilgo P, Puskas JD, et al. (2009) Selective antegrade cerebral perfusion via right axillary artery cannulation reduces morbidity and mortality after proximal aortic surgery. J Thorac Cardivasc Surg 138: 1081-1089.

3. Martin Misfeld, Friedrich W Mohr, Christian D Etz (2013) Best strategy for cerebral protection in arch surgery: Antegrade selective cerebral perfusion and adequate hypothermia. Ann Cardiothorac Surg 2: 331-338.

4. Cefarelli M, Murana G, Surace GG, Castrovinci S, Jafrancesco G, et al. (2017) Elective aortic arch repair: Factors Influencing Neurologic Outcome in 791 patients. Ann Thorac Surg 104: 2016-2023.

5. Kouchoukos NT, Blackstone EH, Hanley FL, Kirklin JK (2013) Barratt-Boyes Cardiac Surgery. (4 $4^{\text {th }}$ edn), elsevier/ saunders, Philadelphia.

6. Mehta RL, Kellum JA, Shah SV, Molitoris BA, Ronco C, et al. (2007) Acute kidney injury network: Report of an initiative to improve outcomes in acute kidney injury. Crit Care 11: 31.

7. Kamenskaya OV, Klinkova AS, Chernyavsky AM, Lomivorotov VV, Meshkov IO, et al. (2017) Deep hypotermic circulatory arrest vs. antegrade cerebral perfusion in cerebral protection during the surgical treatment of chronic dissection of the ascending and arch aorta. J Extra Corpor Technol 49: 16-25.

8. Mohamad Bashir, Matthew Shaw, Michael Desmond, Manoj Kuduvalli, Mark Field, et al. (2013) Cerebral protection in hemi-aortic arch surgery. Ann Cardiothorac Surg 2: 239-244.

9. Di Bartolomeo R, Di Eusanio M, Pacini D, Pagliaro M, Savini C, et al. (2001) Antegrade selective cerebral perfusion during surgery of the thoracic aorta: Risk analysis. Eur $\mathrm{J}$ Cardiothorac Surg 19: 765-770.

10. Dhurandhar V, Parikh R, Saxena A, Vallely MP, Wilson MK, et al. (2016) The aortic root replacement procedure: 12-year experience from the Australian and New Zealand Society of Cardiac and Thoracic Surgeons Database. Heart Lung Circ 25: 1245-1251.

11. Khaladj N, Shrestha M, Meck S, Peterss S, Kamiya H, et al. (2008) Hypothermic circulatory arrest with selective antegrade cerebral perfusion in ascending aortic and aortic arch surgery: A risk factor analysis for adverse outcome in 501 patients. J Thorac Cardiovasc Surg 135: 908-914.

12. Najjar M, Yerebakan H, Sorabella RA, Donovan DJ, Kossar AP, et al. (2015) Acute kidney injury following surgical aortic valve replacement. J Card Surg 30: 631-639.

13. Şahin İşcan, İsmail Yürekli, İhsan Peker, Banu Lafçı, Levent Yılık, et al. (2017) Mitral and aortic valve surgery related acute kidney injury, effecting factors and its long term results. Turk Gogus Kalp Dama 25: 29-35.

14. Antonio Lio, Francesca Nicolò, Emanuele Bovio, Andrea Serrao, Jacob Zeitani, et al. (2016) Total arch versus hemiarch replacement for type $A$ acute aortic dissection: $A$ single centre experience. Tex Heart Inst J 43: 488-495.

15. Yutaka Okita, Hiroaki Miyata, Noboru Motomura, Shinichi Takamoto (2015) A study of brain protection during total arch replacement comparing antegrade cerebral perfusion versus hypothermic circulatory arrest, with or without retrograde cerebral perfusion: Analysis based on the Japan Adult Cardiovascular Surgery Database. J Thorac Cardiovasc Surg 149: S65-S73.

16. Gaudino M, Lau C, Munjal M, Avgerinos D, Girardi LN (2015) 
Contemporary outcomes of surgery for aortic root aneurysms: A propensity-matched comparison of valve-sparing and composite valve graft replacement. J Thorac Cardiovasc Surg 150: 1120-1129.

17. Iscan S, Cakir H, Eygi B, Yurekli I, Donmez K, et al. (2017) Dynamic alterations in cerebral, celiac and renal flows resulting from ascending aorta, subclavian artery and femoral artery cannulations of extracorporeal devices. Perfusion 32: 561-567.

18. Geier A, Kunert A, Albrecht G, Liebold A, Hoenicka M (2017) Influence of cannulation site on carotid perfusion during extracorporeal membrane oxygenation in a compliant human aortic model. Ann Biomed Eng 45: 2281-2297.

19. Demertzis $S$, Tevaearai $H$, Carrel $T$, Vandenberghe $S$ (2011) Aortic flow patterns resulting from right axillary ar- tery cannulation. Interact Cardiovasc and Thorac Surg 12: 973-977.

20. Li B, Hu X, Wang Z (2017) The neurologic protection of unilateral versus bilateral antegrade cerebral perfusion in aortic arch surgery with deep hypothermic circulatory arrest: A study of 77 cases. Int J Surg 40: 8-13.

21. Etz CD, Plestis KA, Kari FA, Silovitz D, Bodian CA, et al. (2008) Axillary cannulation significiantly improves survival and neurologic outcome after atherosclerotic aneurysm repair of the aortic root and ascending aorta. Ann Thorac Surg 86: 441-447.

22. Tiwari KK, Murzi M, Bevilacqua S, Glauber M (2010) Which cannulation (ascending aortic cannulation or peripheral arterial cannulation) is better for acute type A aortic dissection surgery. Interact Cardiovasc Thorac Surg 10: 797-802. 